\title{
A phenotyping platform for transgenic wheat: method and initial results
}

\author{
Raghuveeran Anbalagan ${ }^{1}$, Nataliya Kovalchuk ${ }^{1}$, Boris Parent ${ }^{2}$, Alexander Kovalchuk ${ }^{1}$, \\ Mamoru Okamoto ${ }^{1}$, Ryan Whitford ${ }^{1}$, Stephan M. Haefele $^{1}$ \\ ${ }^{1}$ Australian Centre for Plant Functional Genomics, Adelaide, Australia \\ ${ }^{2}$ French National Institute for Agricultural Research (INRA), Paris, France
}

\begin{abstract}
In recent years, the interest in new technologies for wheat improvement has increased greatly. As methods for conventional and molecular breeding have advanced, more efficient procedures for phenotyping have become a bottleneck. At the Australian Centre for Plant Functional Genetics (ACPFG), a centre for the improvement of wheat and barley's tolerance to environmental stresses, we developed a phenotyping platform for transgenic wheat and barley. After the initial selection of single/low copy number plants in the T0 generation, the $\mathrm{T} 1$ and $\mathrm{T} 2$ generation are screened under competitive growth conditions in large plastic bins. At present, the setup consists of 32 bins which can be used two times a year. All bins are fully equipped with irrigation system and sensors for soil water tension, soil temperature, air temperature, and air humidity, recording data at the time step of 5 minutes. The T1 generation is preferably grown in the summer season (January to June) whereas the T2 generation is evaluated in the main growing season (July to December). The first screening of T1 plants serves to remove off-types (for growth duration, plant height, and other growth anomalies) and to select homozygous plants. In the next step, T2 plants are phenotyped under different soil water and nitrogen regimes in a replicated experimental design in comparison with the wild type and null controls (drought and nitrogen response traits). Plant traits recorded in stage 2 for each individual plant were: tiller number, spike number, empty spikes, plant height, number of seeds, seed weight, and above ground biomass. The soil moisture tension curves of the drought treatments did show a gradual water stress increase over several days to weeks, closely mimicking field conditions. The observed average grain yields per plant were realistic for both well watered and droughted conditions, but seemed still to be too high under the low fertility regime. However, interpreting grain yield data from this setup remained difficult mainly because of the relatively high variability between replications. Further trials are needed to show if this variability can be reduced. We concluded that the new high throughput platform for the testing of transgenic wheat growing in a field-like situation but under controlled conditions was functioning. First season results showed that screening conditions for drought and nutrient use efficiency were as planned and that the platform served its objective. Improvements of the platform to achieve more homogenous results are outlined and the establishment of a digital imaging and analysis system is ongoing. These changes are intended to further increase the diagnostic value of the platform.
\end{abstract}

Keywords: Competitive growth conditions, drought, nutrient use efficiency, phenotyping, wheat 


\section{INTRODUCTION}

With the ongoing population increase outpacing production growth rates of important food crops like rice and wheat, food security returned as a hot topic worldwide. Ever scarcer resources like land and water, and the possibly negative effects of global warming on food production in the near future further contributes to this concern. Of the many possible ways to address these problems, improved crop varieties with e.g., higher yield, improved resource use efficiency or better stress tolerance are always an essential element. But as methods for conventional and molecular breeding have advanced, more efficient procedures for phenotyping have often become a bottleneck. High throughput phenotyping systems are available for the screening of individual plants but the growth behaviour and physical characters of single plants usually grown in small pots are not necessarily reproduced in the field (Roy et al., 2011). One crucial factor causing this difference is that under field conditions, plants are subject to additional stresses of having to compete with their neighbours and weeds for light, water and nutrients present in the soil. We were therefore interested in developing a new phenotyping platform for transgenic wheat and barley, allowing a more realistic screening for tolerance to environmental stresses (e.g., drought) and improved resource use efficiency. Thus, our objectives were to 1) develop an experimental setup where transgenic wheat and barley can be evaluated under competition with other plants, comparable to a situation a crop stand experiences, whilst under specific abiotic stress conditions, 2) to establish adequate testing methods and procedures ensuring the functionality of the phenotypic pipeline and 3) to determine the efficiency of this setup for the evaluation of transgenic plants.

\section{MATERIAL AND METHODS}

\section{Phenotyping stages in the evaluation of transgenic wheat germplasm}

Transgenic wheat plants produced with the particle bombardment method (but soon to be replaced by Agrobacterium transformation) undergo the following testing and multiplication procedure. Leaf samples from individual plants in the T0 generation, in-vitro generated in Petri dishes, were tested for copy number of the construct based on genomic DNA using quantitative Polymerase Chain Reaction (PCR) and/or Southern blotting. RNA extracted from leaves was used to test for the expression level of the transgene. Plants were then transferred into pots, and grains from T0 plants with single or low copy number (T1) and a defined expression level of the transgene were selected for the Stage 1 phenotyping.

The bin setup for Stage 1 phenotyping is shown in Figure 1a. Because of limited space, one bin was used per construct. In each bin, plants were grown in a raster with 10 rows of 8 plants each in addition to the border row (planted with the variety Gladius). Of these 10 rows, one was planted with the wild type used for transformation, and each of the remaining nine rows represented plants from separate transgenic events but with the same transgene. T1 seeds were pre-germinated on moist paper in a Petri dish at room temperature for 3 days and then transplanted. Soil water tension was kept $<50 \mathrm{kPa}$ by regular watering. After 7 days, plants were scored for first leaf emergence and pre-germinated wild type plants were sown to replace empty positions. At flowering, all plants differing from the wild type by more than \pm 2 weeks in flowering date were removed from the further analysis. Plants at flowering, which were more than $10 \mathrm{~cm}$ higher or shorter than the wild type were also removed from further analysis. And finally, all plants with phenotypes not related to the transgene (off types) were also removed. At maturity, when shoot tissues were completely dry, plants were harvested and individually bagged in paper bags. Parameters measured were spike number, seed number, and seed weight. After data recording, the seeds were bagged, labelled and stored in a PC2 seed storage facility. Other plant material was autoclaved prior to disposal.

Because seed of the T0 plants segregates with respect to the transgene, T1 plants in Stage 1 are either homozygous, heterozygous, or null (i.e., do not carry the transgene). To determine homozygous plants, 12 seeds from each T1 plant in Stage 1 were germinated on a Petri dish, leaf samples were taken and individually analysed for the transgene by quantitative PCR. Homozygosity was assumed if all 12 grains carried the transgene, and the remaining seed from that plant was then advanced to Stage 2 testing. The setup for Stage 2 phenotyping and the testing procedure is shown in Figure 1a and b. Each bin has 10 subplots, flanked by a border row (var. Gladius) on each short side of the bin. No border row was deemed necessary on the long side of the bin because all sub-plots were the same and to maximize the available space per bin. The 10 subplots were then used to screen 3 transgenic T2 lines (TG1-3, theoretically homozygous, having single or low copy number, and showing expression of the transgene), the wild type (WT) used for transformation, and one Null control (N), all with two replications. At maturity, the same procedures as described above were used for harvesting, seed storage, and biomass disposal. Plant parameters recorded in stage 2 for each 
individual plant were: tiller number, spike number, empty spikes, plant height, total number of seeds, total seed weight, and total above ground biomass.

Stage 1. Off-type removal:

1 Bin: 80 plants

9 events, 1 WT control

8 plants per event or WT

1 bin per construct

$120 \mathrm{~cm}$

Stage 2. Testing of promising events:

1 Bin: 10 subplots, 8 plants per subplot

3 TG lines, 1 WT control, 1 Null control $\times 2$ reps

Randomised block design

2 bins for 3 events if stress is applied

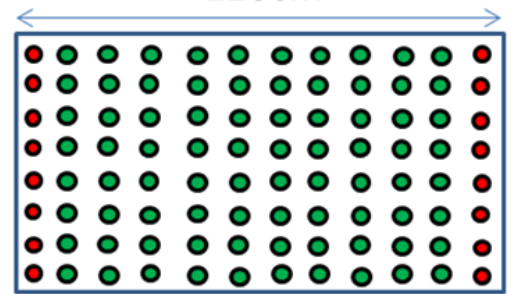

$120 \mathrm{~cm}$

(a)

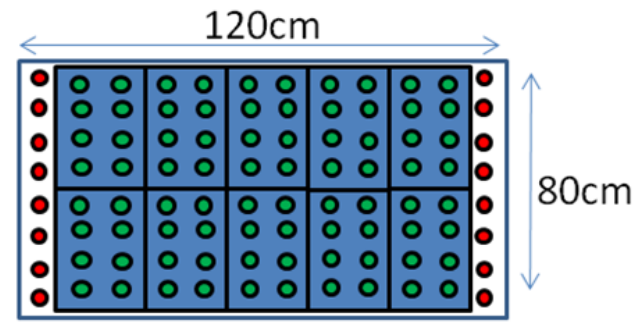

(b)

T0: $50+$ events

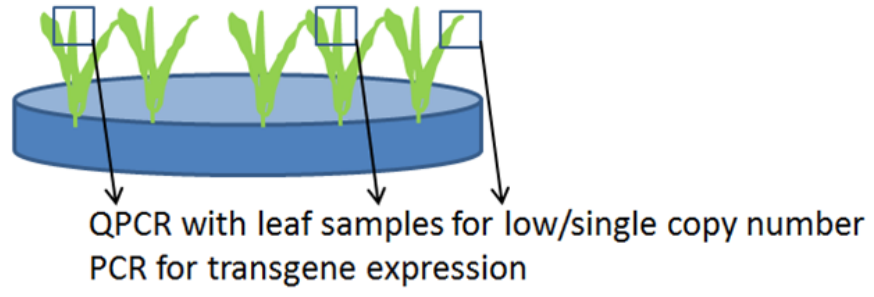

- If single event is determined, the segregation (1:2:1) should produce $1 / 4$ homozygous grains with the transgene.

- T1: reduced to 9 events with low/single copy number. The T1 is grown in bins and off-types (mainly flowering date and plant height) are removed (o).

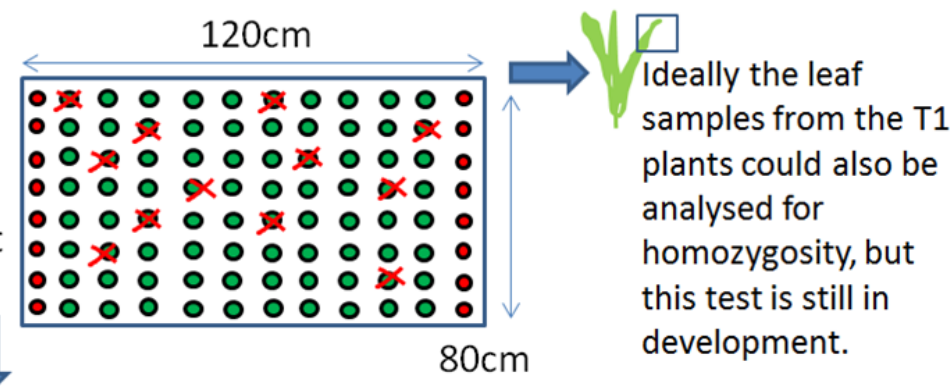

Alternatively, 12 grains from each T1 plant are analysed for the transgene. If all plants from a single T1 contain the transgene its other grains are most likely homozygous

\section{T2 testing}

Figure 1: details of the setup of transgenic plants in the bins for Stage 1 and 2 testing (a) and an overview of the testing procedure to produce homozygous plants for the Stage 2 testing (b).

\section{Experimental design}

The plastic bins used for the Stage 1 and 2 experiments had internal dimensions of $1.12 \mathrm{~m}$ length, $0.76 \mathrm{~m}$ width, and $0.60 \mathrm{~m}$ depth. A double floor was installed at the bottom of the bins to ensure good drainage, reducing the internal depth to $0.45 \mathrm{~m}$. The bins were then filled with soil prepared by mixing 2 parts of clay loam (Clay \& Minerals PTY Ltd, Golden Grove, Adelaide) with 1 part of Coco Peat (mixed by SARDI from Coco Peat, Waikerie sand, and various fertilizers) and 1 part of UC mix (mixed by SARDI from Waikerie sand, peatmoss and various fertilizers). This soil mix was heat sterilized and contained already a basal rate of 
$\mathrm{N}$ fertilizer (Mini OSMOCOTE slow release fertilizer + selected other soil amendments). A total of 32 bins thus prepared were installed in a greenhouse (SARDI plant research centre, Waite campus, Adelaide, Australia), certified as Physical Containment Level 2 (PC2) Laboratory according to the requirements of the Office of the Gene Technology Regulator (OGTR, 2013). The preferred sowing date for all bins is midAugust and mid-January but delays of up to a few weeks can occur and very late seeding (11 $1^{\text {th }}$ October 2012) was used in the first experimental season described here. Only daylight is provided in the winter season (starting mid-August), but in the summer season (starting late January) we applied extra light in the morning and evening to accelerate ripening towards the end of the growing season which takes place over a period of 3-4 weeks. Air-conditioning in the greenhouses restricted temperature fluctuations, maintaining it between $15^{\circ} \mathrm{C}$ and $30^{\circ} \mathrm{C}$ all year round, with air humidity ranging between 40 and $80 \%$. Irrigation was provided through a drip irrigation system and regulated according to the water treatments. Eight soil water tensiometers (gypsum blocks) were installed at 0.1 and $0.3 \mathrm{~m}$ depths and connected to a data logger for continuous monitoring of soil water tension. For all trials, soil and air temperature, relative air humidity, and soil moisture tension were automatically monitored with data loggers. Representative soil samples were analysed for standard soil parameters (results not yet available).

Transgenic wheat material was tested in this setup for two characteristics: Drought Tolerance (DT) and Nutrient Use Efficiency (NUE). For drought testing in Stage 2, each set of entries to be tested (TG1-3, WT, $\mathrm{N})$ were planted according to the layout shown in Figure 1a in two bins. One bin was watered normally (WN) and the other bin was drought stressed periodically (WS). The same setup was used for TG4-6. Drought was imposed at anthesis and early grain filling, when wheat is sensitive to water deficit (Stone and Nicholas, 1995). All bins used for drought tolerance testing received the standard fertilizer rate incorporated in the soil mix. To test germplasm for NUE, a mixed experimental setup combining different fertilizer rates and drought treatments was used. Two bins received the standard nitrogen rate but one was irrigated normally (NNWN) and the other drought stressed (NNWS). Again, water stress was imposed at anthesis and grain filling. Two additional bins were well-watered but received a lower than normal (NLWN) and a higher than normal nitrogen rate (NHWN). The same set of entries was used for all four bins using the stage 2 layout described in Figure 1a. Transgenic plants tested were based on two different parents, Gladius and Frame. The entry set for NUE testing included the Gladius WT, Nulls of both parents, and two different transgenic plants (TG7-8). In all cases, the actual genes used are under IP restrictions and cannot currently be named.
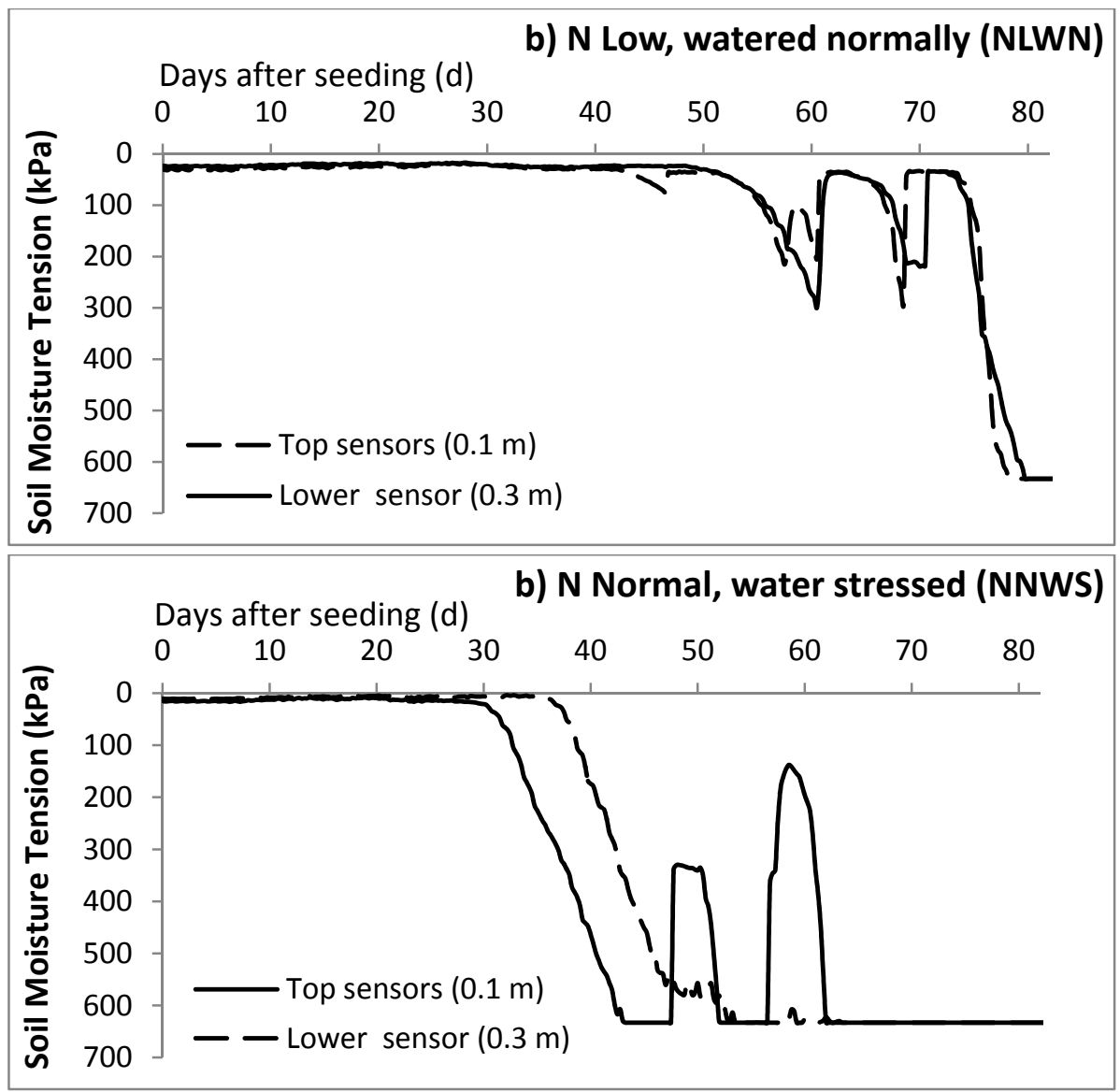

Figure 2:

Representative changes in soil water tension over time in the NUE experiment. 


\section{RESULTS AND DISCUSSION}

In all water stressed treatments, irrigation was withheld at 30 days after seeding for 18 days, then re-irrigated at 48 and 58 days after seeding (see the water stress treatment in Figure 2). For these late irrigations, the amount was intended to replicate a $25 \mathrm{~mm}$ light rain (corresponding to 21.3 liter of water per bin) and dripped in over 17.6 minutes (calculated based on the flow rate in the drip lines). An example of the soil water tension changes during the whole season is shown in Figure 2. It shows that the soil water tension increased slowly during the first stress period, but more rapidly after the two re-watering events. Re-watering at 48 and 58 days after seeding mimicked two rain events from a typical south-Australian or Mediterranean climate. Plant symptoms of leaf rolling and wilting were detected from more than $633 \mathrm{kPa}$ soil water tension onwards, however, soil moisture tensions beyond $633 \mathrm{kPa}$ could not be measured by the sensors used.

Flag leaf water potential measured at leaf wilting point, using a Scholander pressure chamber (Delta XXX), reached $1500 \mathrm{kPa}$, indicating a high stress level which was confirmed by the plant measurements (see data below). The change in soil water tension shows that drought stress did occur at anthesis (between 47 to 54 days after seeding), and throughout grain filling (up to 82 days after seeding), as planned. Screening under such stress conditions is considered to be an adequate strategy for wheat improvement in Mediterranean climates with late season drought (Araus et al., 2002). In general, the effect of drought stress depends also on the nutrient supply but significant dry matter losses in well-fertilized soil were reported for water tensions above $200 \mathrm{kPa}$ (Plaut, 1973). All germplasm tested was mature after 82 days and only ripening/drying-off thereafter. Gladius is a short-duration variety but the late planting experienced in this study even shortened its normal duration.

Table 1: Water treatment effects across all entries and replications for selected characteristics at harvest and the two entry sets.

Treatment

\begin{tabular}{|c|c|c|c|c|c|c|c|c|c|c|}
\hline $\begin{array}{l}\text { Entry } \\
\text { set }\end{array}$ & $\begin{array}{l}\text { Water } \\
\text { status }\end{array}$ & $\begin{array}{l}\text { Tiller } \\
\text { num. } \\
\text { (n) }\end{array}$ & $\begin{array}{l}\text { Spike } \\
\text { num. } \\
\text { (n) }\end{array}$ & $\begin{array}{l}\text { Empty } \\
\text { spike } \\
\text { num. } \\
\text { (n) }\end{array}$ & $\begin{array}{l}\text { Plant } \\
\text { height } \\
(\mathrm{cm})\end{array}$ & $\begin{array}{l}\text { Total } \\
\text { grain } \\
\text { num. } \\
\text { (n) }\end{array}$ & $\begin{array}{l}\text { Total } \\
\text { grain } \\
\text { weight } \\
(\mathrm{g})\end{array}$ & $\begin{array}{c}\text { Total } \\
\text { dry } \\
\text { weight } \\
\text { (g) }\end{array}$ & $\begin{array}{c}\text { Harvest } \\
\text { Index } \\
(-)\end{array}$ & $\begin{array}{c}1000 \\
\text { grain } \\
\text { weight } \\
(\mathrm{g})\end{array}$ \\
\hline 1 & WN & 7.1 & 7.1 & 0.0 & 75.3 & 239 & 5.9 & 19.6 & 0.30 & 24.6 \\
\hline 1 & WS & 8.3 & 1.8 & 0.1 & 44.7 & 29 & 0.8 & 6.9 & 0.12 & 29.5 \\
\hline 2 & $\mathrm{WN}$ & 10.5 & 7.3 & 0.2 & 74.6 & 233 & 7.4 & 20.8 & 0.36 & 32.1 \\
\hline 2 & WS & 9.9 & 3.9 & 0.8 & 50.2 & 71 & 1.6 & 10.9 & 0.14 & 24.0 \\
\hline
\end{tabular}

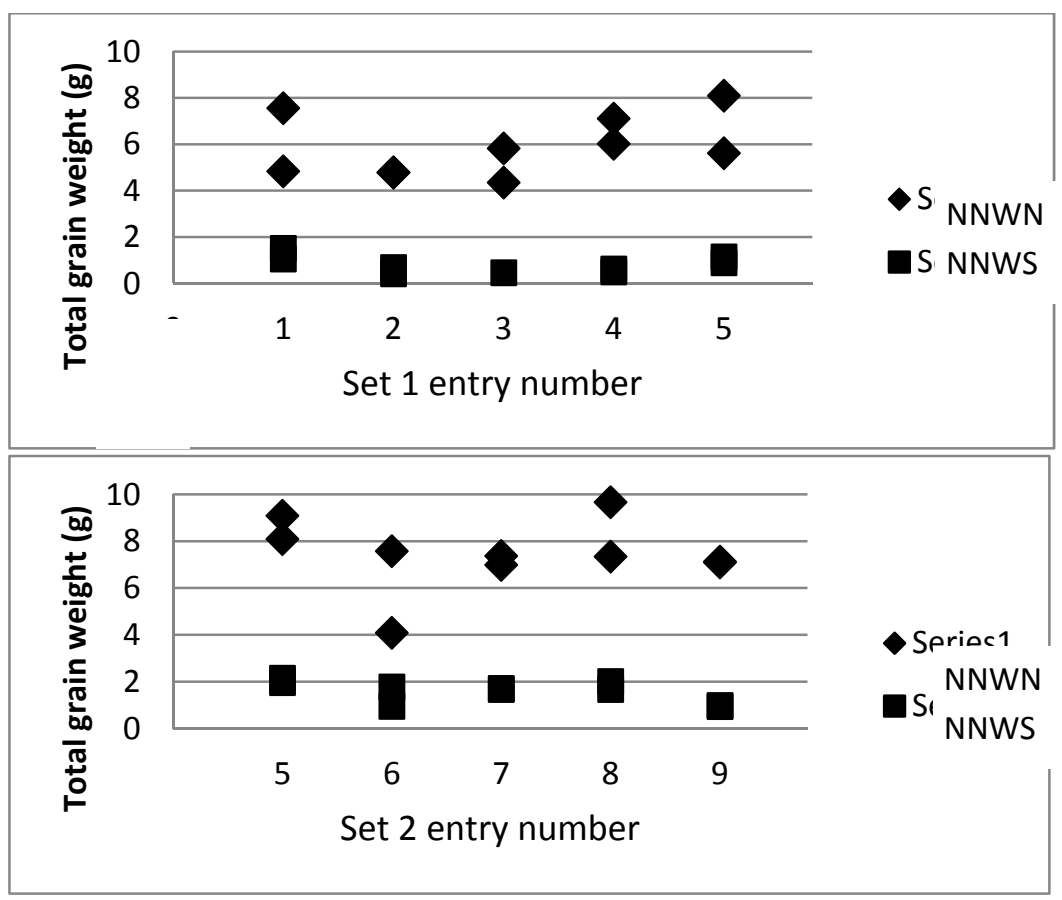

\section{Set 1 Name}

$\begin{array}{ll}1 & \text { Gladius WT } \\ 2 & \text { Gladius Null } \\ 3 & \text { Gladius TG1 } \\ 4 & \text { Gladius TG2 } \\ 5 & \text { Gladius TG3 }\end{array}$

Set 2

5 Gladius WT

6 Gladius Null

7 Gladius TG4

8 Gladius TG5

9 Gladius TG6

Figure 3: Total grain weight for each replication of all entries and two treatments used to determine drought tolerance. 
The average effect of the treatments in the drought stress experiment is shown in Table 1. In both entry sets tested applied drought stress significantly decreased spike number, plant height, total grain number, total grain weight, total dry matter, and harvest index, indicating considerable water stress from the stem elongation stage of development onwards. No effect was observed on tiller numbers because they were intiated before the onset of stress. Empty spikes with no grains were observed as a consequence of the drought stress, and increased with increasing magnitude of the stress. No clear effect could be found for the average 1000 grain weight because it increased in set 1 and decreased in set 2 .

As an indicator for the drought stress effects on individual entries, the mean total grain weight per plant is shown in Figure 3. In both sets and both treatments, the Gladius wild type performed better than the Null plants, which is a frequent observation and likely consequence of the transformation and tissue culture process. In the drought-stressed treatments, none of the entries seemed to have any advantage compared to the wild type and the Nulls, but in well-watered treatments, Gladius TG2, 3 and 5 were performing better than their respective Nulls, and TG2 and 3 had a higher average grain yield per plant than the Gladius wildtype. A full statistical analysis is available but not presented here.

Table 2: NUE treatment effects across all entries and replications for selected characteristics at harvest.

Treatment

Plant

N- Water Tiller Spike Empty spike Plant Total Total level status num. num. num. height

Average values per plant

\begin{tabular}{llcccccccccc} 
& & & & & & & num. & weight & weight & Index & weight \\
& & $(\mathrm{n})$ & $(\mathrm{n})$ & $(\mathrm{n})$ & $(\mathrm{cm})$ & $(\mathrm{n})$ & $(\mathrm{g})$ & $(\mathrm{g})$ & $(-)$ & $(\mathrm{g})$ \\
\hline $\mathrm{NN}$ & $\mathrm{WN}$ & 5.5 & 5.5 & 0.0 & 77.7 & 192 & 6.6 & 18.9 & 0.35 & 34.2 \\
$\mathrm{NN}$ & $\mathrm{WS}$ & 4.3 & 1.9 & 0.1 & 41.7 & 26 & 0.9 & 7.4 & 0.12 & 32.1 \\
$\mathrm{NH}$ & $\mathrm{WN}$ & 4.2 & 4.2 & 0.0 & 81.8 & 169 & 6.3 & 17.3 & 0.36 & 36.9 \\
$\mathrm{NL}$ & $\mathrm{WN}$ & 3.8 & 3.8 & 0.0 & 79.0 & 141 & 5.3 & 14.7 & 0.36 & 37.5 \\
\hline
\end{tabular}

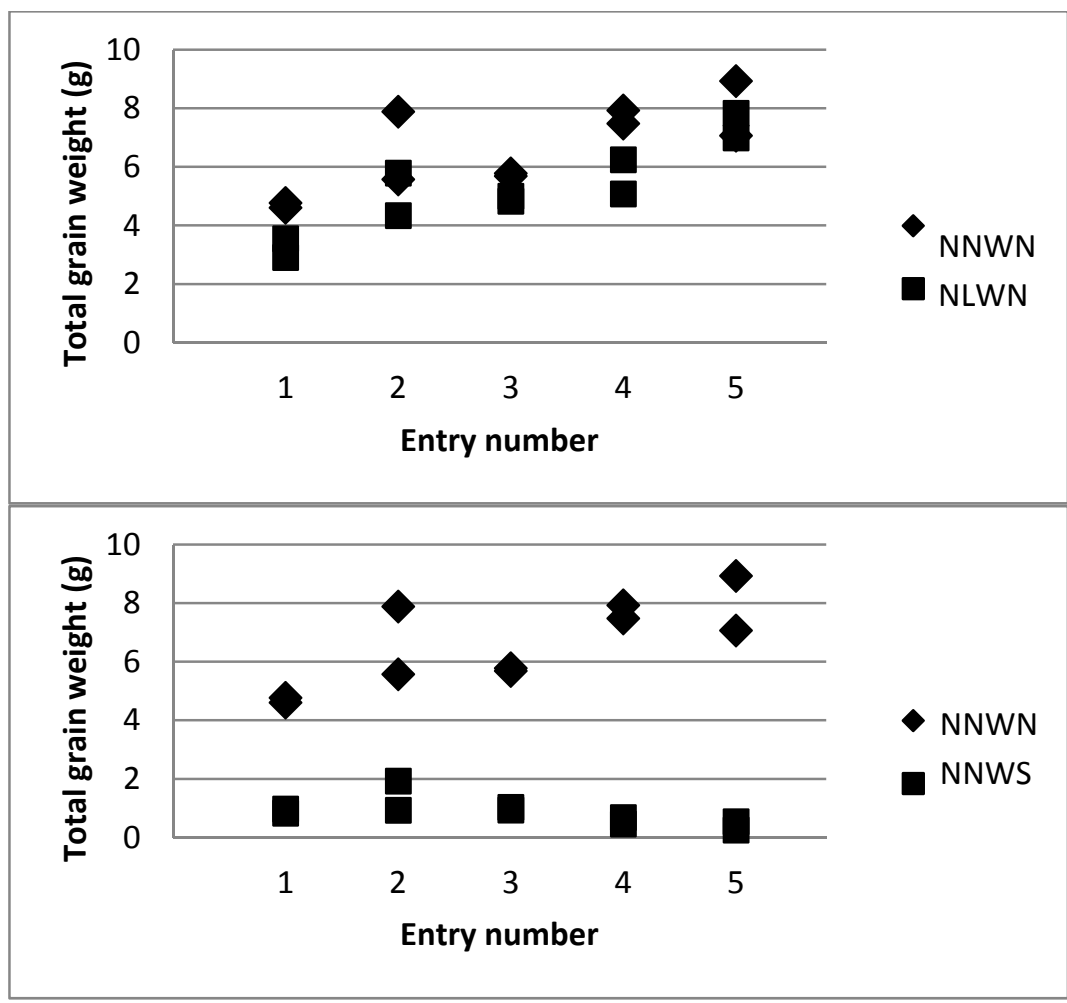

Figure 4: Total grain weight for each replication of all entries and three treatments used to determine NUE. 
The average treatment effect in the NUE experiment is shown in Table 2. Again, the drought treatment NNWS revealed a clear effect on plant growth, yield components, and total biomass across all tested lines. The low $\mathrm{N}$ treatment (NLWN) significantly reduced tiller numbers, spikes, and grains, and a lower total grain weight and biomass were also observed. However, the high $\mathrm{N}$ treatment (NHWN) had no significant effect in comparison with normal $\mathrm{N}$ rate treatment $(\mathrm{NNWN})$ and performed even slightly poorer. This indicates that the $\mathrm{N}$ rate in the normal $\mathrm{N}$ treatment is already very high and not limiting to plant growth. Rather than a higher $\mathrm{N}$ treatment, a treatment without any $\mathrm{N}$ application might be a better option for further testing the entries under low $\mathrm{N}$ conditions. This would be more typical for most wheat environments in Australia and many wheat environments worldwide (Hooper and Johnson, 1999).

The treatment effect on individual entries is shown in Figure 4 but only for three treatments with a clear effect (thus, NHWN is not shown). Again Gladius wild type performs considerably better than Gladius Null. None of these entries had grain yield advantage relative to Gladius wild type under drought stress. Gladius TG7 and Frame TG8, however, performed better than their respective Nulls, with both transgenic lines performing better under low $\mathrm{N}$ conditions (NLWN). Again, a full statistical analysis is not presented here, and we are still in the process to determine the most appropriate methodology for data analysis (mean versus median values, determine which other observed characteristics apart from grain yield could be used to evaluate the entries, etc.).

Since these were the initial trials for our new transgenic wheat testing setup, the trials will be repeated. However, the data presented in Figures 3 and 4 does allow us to draw some preliminary conclusions regarding the functionality of this platform. The soil moisture tension curves of the drought treatments do show a gradual water stress increase over several days to weeks, closely mimicking field conditions. Testing at full and limited water supplies ensures that selected genotypes would perform well in both conditions. But it needs to be decided if testing at medium stress levels would be the better choice (Araus et al., 2002). The observed average grain yields per plant are realistic (as an estimate, the values in g per plant can be converted into tons per ha based on a $10 \times 10 \mathrm{~cm}$ spacing) for both well watered and droughted conditions, but seem still to be too high under the low fertility regime (a target of $3 \mathrm{~g}$ grain per plant might be more adequate). Interpreting grain yield data from this setup remains difficult for two main reasons: one is the relatively high variability between replications. Further trials are needed to show if this variability can be reduced. If not, options would be to increase the numbers of replications (which would reduce the throughput capacity), or to use the results from the bins as a first indication only, which then has to be verified in field trials (advanced T3 testing). Including advanced imaging methods (Kumar et al., 2002, 2003) is ongoing and will allow a continuous and much more precise monitoring of plant performance. The other main question raised by these results comes from the often superior performance of the wild type versus the transgenic lines. This (possibly pleiotropic) effect has also been described for rice (Xiao et al., 2009) but was absent in a recent study by Saint Pierre et al. (2012). Thus, optimizing the transformation process and tissue culture could possibly reduce or even remove this effect.

\section{REFERENCES}

Araus, J.L., Slafer, G.A., Reynolds, M.P., Royo, C. (2002). Plant breeding and drought in C3 cereals: what should we breed for? Annals of Botany 89, 925-940.

Hooper, D.U., Johnson, L. (1999). Nitrogen limitation in dryland ecosystems: responses to geographical and temporal variation in precipitation. Biogeochemistry 46, 247-293.

Kumar, P., Ranganath, S., Weimin, H., 2003. Queue based Fast Background Modelling and Fast Hysteresis Thresholding for Better Foreground Segmentation. In: Proceedings of 2003 Joint Conference of the Fourth International Conference on Information Communications and Signal Processing, pp. 743-747.

Kumar, P., Sengupta, K., Lee, A., Ranganath, S., 2002. A Comparative Study of Different Color Spaces for Foreground and Shadow Detection for Traffic Monitoring System. In: Proceedings of the IEEE 5th International Conference on Intelligent Transportation Systems, pp. 100-105.

OGTR (2013). Guidelines for the Certification of Physical Containment Facilities: PC2 plant facility Ver. 3.2. Available at http://www.ogtr.gov.au/internet/ogtr/ publishing.nsf/Content/certifications-1.

Plaut, Z. (1973). The effect of soil moisture tension and nitrogen supply on nitrate reduction and accumulation in wheat seedlings. Plant and Soil, 38:81-94.

Roy, S.J., Tucker, E., Tester, M. (2011). Genetic analysis of abiotic stress tolerance in crop plants. Current Opinion in Plant Biology 14: 232-239.

Saint Pierre, C., Crossa, J.L., Bonnett, D., Yamaguchi-Shinozaki, K., Reynolds, M.P. (2012). Phenotyping transgenic wheat for drought resistance. Journal of Experimental Botany, p. 1-10. doi:10.1093/jxb/err385.

Stone, P.J., Nicholas, M.E. (1995). Comparison of sudden heat stress with gradual exposure to high temperature during grain filling in two wheat varieties differing in heat tolerance. I. Grain growth. Aust. J. Plant Physiol., 22: 935-944.

Xiao B, Chen X, Xiang C, Tang N, Zhang Q, Xiong L. (2009). Evaluation of seven function-known candidate genes for their effects on improving drought resistance of transgenic rice under field conditions. Molecular Plant 2, $73-83$. 